\title{
Formatos de intercambio, compatibilidad y normalización
}

\section{RESUMEN}

Se con cep tua li za lo inhe ren te a un for ma to de in ter cam bio y se pun tua li zan los con cep tos de nor ma li za ción y de trans fe ren cia de da tos en el con tex to bi blio te ca rio, se plan tea el pa pel de las nor mas en lo que con cier ne al in tercambiodere gis tros bibliográficos le gibles por computadora.

Asimismo son presentados aspectos relevantes sobre diferentes formatos para intercambiar información bibliográfica automatizada, como lo son; MARC, UNIMARC, IN TER MARC, y el CCF, en tre otros. Se des cri be su evo lu ción y apli ca ción, asi como el pa pel que han asumido en la normalización de información bibliográfica para su intercambio.

Enbase con diferen tes opiniones de es pecia lis tas en sis te mas au to ma ti za dos parabiblio te cas, se pre sen tan pun tos de vis ta de ri va dos de la aplicación de esos formatos en diferentes contextos bibliotecarios a un marco internacional.

\section{SUMMARY}

The essen tial is con cep tua li zed in a for mat of in ter chan ge. The con cepts of nor ma li zation and of exchange of data in a library context are em pha si zed. The role of norms in a li brary con text in that wich con cerns the in ter change of ma chi ne rea da ble bib-lio gra phic re cording is set forth. Likewise different formats present relevant aspects for interchange of automized bibliographic information such as MARC, UNIMARC, INTERMARC and CCF among others.

On the ba sis of opi nions of spe cia lists in au to mi zed sys tems for li bra ries, points of view are pre sen ted, de ri ved from the appli ca tion of these formats in differing library contexts on a international level.

\section{Panorama General}

Un formato de intercambio de información bibliográfica automatizada se refie re al mé to do de or ga ni zar la in for ma ción de tal mane ra que las áreas ca ta lo grá fi cas pue dan seriden ti fi ca das, así como las parti cu la ri da des de co di fi ca ción que se se ña len en cada área com po nen te de un re gis tro bi blio grá fi co. De fi nir con pre cisión los elementos constitutivos de un formato, representa un profundo análisis de la información que requiera ser almacenada, re cu pe ra da o in ter cam bia da, de bi do que a par tir de la es tructura del formato se pue den de fi nir los ni ve les de ex plo ta ción de la información almacenada en bases de datos.

Lanormalización en el contexto del in ter cambiode re gis tros bibliográficos también es de fundamental importancia, ya que a tra vés de ella, se pue den ma ne jar el con jun to de con di cio nes que debe satisfacer el producto de intercambio.

"Las normas son especificaciones técnicas que contienen un conjun to de con di cio nes a ser cum pli das. Pue den ser pro du cidas por empresas, asociaciones, ministerios y organismos nacionales o regionales de normalización".
Sea cual fue re el ori gen de las nor mas, és tas son de gran uti li dad ya que per mi ten co or di nar, simplificary unifor mar criterios con la ven ta ja de ob te ner ma yo res pro duc tos a me nor cos to y con rapidez.

Con relación al ámbito documental, se han realizado diferentes esfuerzos con el fin de establecer procedimientos normalizados para el intercambio de información bibliográfica en forma legible a través de computadora. Por información bibliográfica se entiende, entre otros materiales: referencias bibliográficas de monografías, publicaciones periódicas, materiales audiovisuales, artículos de revistas, indización de diferentes documentos, anotaciones de es que mas declasificación, términos procedentes de sis te mas basa dos en len gua je con tro la do talcomoTesaurus, o esquemas de encabezamientos de materia.

Esos es fuer zos han per mi ti do que en la ac tua li dad, exis tan di ferentes for ma tos paraelintercambiodeinformación bibliográfica automatizada, cuya premisa consiste en normalizar para compartir la información. 
El pri mer es fuer zo de co ber tu ra in ter na cio nal re fe ri do a la ge nera ción de un for ma to de in ter cam bio, sur gió en la Bi blio te ca del Con gre so de los Es ta dos Uni dos. Allá se creó el for ma to MARC, en 1966 y des pués de su en sa yo ex pe ri men tal se me jo ró en co ope ra ción con la Bi blio te ca Na cio nal Bri tá ni ca (BNB). Como resul ta do de esto na ció lo que fue el pri mer for ma to de in ter cam bio internacionalestructuradosobrebases metodológicasconsistentes y normalizadas.

No me nos im por tan tes fue ron los es fuer zos de los for ma tos predecesores de los formatos MARC tales como el: UNIMARC, INTERMAC, MARKOF, el Manual de Referencias del UNISIST, y el CCF, entre otros.

En la actualidad se puede apreciar que un gran porcentaje de la comunidad bibliotecaria internacional, se encuentra utilizando el for ma to MARC y los cen tros de ser vi cios de re sú me nes e in dización, uti li zan el for ma to con te ni do en el Ma nualde Re fe ren cia UNISIST, asi mis mobibliotecas na cionales de diferen tes países, utilizan el for ma to UNIMARC y recien te men te em pie zan a usar el formato CCF.

Al exis tir una se pa ra ción real en tre la in for ma ción pro ce sa da en bi blio te cas y cen tros de do cu men ta ción y el uso de va rios for matos, el in ter cam bio de in for ma ción se di fi cul ta, pero exis te la posi bi li dad de ge ne rar pro gra mas de cóm pu to que ha gan po sible la compatibilidad de formatos, sin em bar go, tam bién es cier to que para ge ne rar esos pro gra mas se re quie re de tiem po y de re cur sos económicos, que generalmente son altos.

La pro lifera ción de for ma tos de in ter cam bio de re gis tros, no obstante que la mayoría son derivados del mismo origen, ha sido causa de preocupación durante muchos años.

Duchesne ha señalado que:

"El resultado será una babel de métodos nacionales que pronto resultará inmanejable en una situación dinámica."

A pe sar de los avan ces elec tró ni cos que se han lo gra do, esta observación de Duchesne hecha en 1972, podría considerarse vigente ya que el intercambio de registros entre unidades de información se sigue dificultando.

El in ter cam bio de re gis tros bi blio grá fi cos por par te de agen cias internacionales de intercambio se ha realizadoprincipalmente a tra vés de cin ta mag né ti ca y la nor ma in ter na cio nal que rige el intercambio es la norma ISO 2709.

Esta nor ma de fi ne la es truc tu ra ge ne ral que de ben guar dar los regis tros para su in tercambio. Sin em bargo, no de fi ne el con te ni do de los re gis tros ni los sig nifica dos de eti que tas, in di ca do res y códigos de subcampo.

Boi ko y Polozhentse va han se ña la do, la nor ma ISO, como exis te ac tual men te, cu bre la es truc tu ra ge ne ral del re gis tro de in for mación y co di fi ca el uso de cua tro ti pos di fe ren tes de es truc tu ras de re gis tro. No obs tan te: "no tra ta los as pec tos se mán ti cos, el sig ni- fi ca do de las eti que tas, los me dios para for mar la des crip ción bibliográfica. La norma no fue suficiente para resolver los problemas de compatibilidad de archivos de bases de datos." A pesar de esto la norma ISO 2709, sigue vigente.

Una varie dad de normas in ternacionaleses tán directa menterelacionadas con los elementos de la información y su representación, tales como las normas relacionadas con la transliteración de alfabetos no romanos. Los llamados Principios de París, que en la ac tua li dad se re fle jan en la ma yo ría de los có di gos de ca talogación, han ayudado a promover la normalización de manera prio ri ta ria en la elec ción de los ele men tos de in for ma ción ca ta lográfica que serán almacenados.

Es evi den te no tar que nor ma li za ción y com pa ti bi li dad son in separables en el mar co del in ter cam bio de re gis tros biblio gráficos. Normalización y compatibilidad en este contexto se deben observaren diferen tes ni ve les, asíte ne mos que un for ma to de in tercambio está involucrado íntimamente a distintos niveles; con aspectosinformáticos, bibliotecarios y normativosdeintercambio.

La experiencia internacional en el intercambio de registros, ha mostrado que la compatibilidad total, hasta el momento, no ha sido posible de alcanzar, debido fundamentalmente a que no es co mún que va rias ba ses de da tos se en cuen tren uti li zan do el mismo equi po de cóm pu to, los mis mos pro gra mas de com pu ta do ra, el mismo formato de intercambio y las mismas normas de descripción catalográfica, así como el mismo nivel de descripción. Sólo si sucede esto, se podría hablar de compatilidad absoluta.

La integración de las bases de datos exige la normalización de for ma tos de in ter cam bio, y más es tric ta men te de uno solo de manera que, por lo que respecta a la computadora, sólo haya que procesar un formato único.

Boiko y Polozhentseva han señalado:

"Un for ma to lle va a la prác ti ca un con jun to de de ci sio nes se mánticas y formales relativas al conjunto de elementos utilizados para describir un documento de tipo específico."

Cuan do se crean pro gra mas para in ter cam biar los ar chi vos de infor ma ción, de be mos ana li zar, a la vez, las ca rac te rís ti cas es tructurales y semánticas de la descripción de un documento y encontrar los medios para convertir una estructura semántica a otra. Los formatos del sistema en estudio serán compatibles si podemos compilar un programa de conversión que nos permita ex traer de los ar chi vos las carac te rís ti cas bi blio gráficas y se mánticas necesarias para la re cu pe ra ción en un sis te ma de ter mi na do. Como se ha men cio na do, el proble ma de la com pa ti bi li dad de registros bibliográficos para su intercambio, ha sido un factor de preocupación en trelaco mu nidadbibliotecariain ter nacional, no obstante ha sido ella mis ma quien ha ge ne ra do con fu sio nes e inhibición en el intercambio debido fundamentalmente a la generación de diversos formatos de intercambio. 
En la literatura sobre formatos de intercambio, se afirma que la re dac ción de progra mas de cóm pu to de con ver sión, re pre sen ta la so lu ción de la com pa ti bi li dad, lo cual en cier ta me di da es ve rídico; sin embargo, parece que no se tiene conciencia de que para poder redactar los programas de compatibilidad se requiere de un análi sis pre vio y profun do so bre los por me no res de los for matos que se requiera hacer compatibles y esto implica tiempo de investigación que generalmente no es de una sola persona sino más bien de un grupomultidisciplinario.El otro as pec to que no se especificaa profundidad es el que se refiere al tiempo que se re quie re para rea li zar el di se ño de los pro gra mas de con ver sión o de com pa ti bi lidad, que en ge ne ral pue de ser un lap so con si de rable ya que hasta la actualidad se tiene muy poca experiencia en este sentido y quienes han te ni do la ex pe rien cia son profe sionales que laboran para una gran unidad de información o son miembros de alguna comisión de reconocidas asociaciones de bibliotecarios, los que además cuentan con suficientes bases eco nó mi cas como para fi nan ciar un pro yec to de con ver sión o de compatibilidad de formatos de intercambio.

A pesar de haberse generado estudios por reconocidos especialistas en el ámbito bibliotecario, no se ha logrado hasta la fecha ge ne rar un pa que te de pro gra mas que per mi tan la con ver sión auto má ti ca de re gis tros en un mar co in ter na cio nal; más bien lo que has ta el mo men to se ha lo gra do es la com pa ti bi li dad de re gis tros a nivel nacional o entre grandes unidades de información internacional que bajo convenios, observan normas técnicas que les facilitan la compatibilidad en el intercambio.

Lacompatibilidad en el intercambio de registros debe observarse en distintos niveles. El nivel más alto de la compatibilidad sólo pue de lo grar se si se uti li zan los mis mos cri te rios téc ni cos, es de cir, la mis ma es truc tu ra de re gis tros, el mis mo for ma to que defina designadoresdecontenido, y las mis mas re glas para la descripción catalográfica.

Por ejemplo, el servicio de intercambio USMARC está basado entre otros, en los siguientes elementos: estructura conforme a MARC formats for bibliografic data, estructura del registro en ISO 2709, des crip ción ca ta lo grá fi ca con base a AACR2, el có digo de máquina utilizado es el ALA Extended ASSCII, y cinta magnética de siete y nueve canales

Estos datos técnicos determinan que las bases de datos que deseen in tercam biarre gis tros con la Biblio te ca del Con gre so de los Estados Unidos, deberán observar estos criterios técnicos.

El formato LCMARC, es compatible con los formatos MARC de bi do a que ob ser va las nor mas es pe ci fi ca das por éste. Sin embar go, es im por tan te acla rar que los for ma tos MARC, no son utili za dos en su to ta li dad sino más bien la Biblio te ca del Con gre so, utiliza en su formato LCMARC, las etiquetas, códigos de subcam po e in di ca do res que le son de utili dad para proce sar su in formación, lo cual es totalmente válido, ya que no tendría sentido que usa ra cam pos que sólo le son de uti li dad a otras bi blio te cas; asimismo, las Reglas de Catalogación Angloamericanas 2, adop tadas poresabiblioteca, tam po co son observadas de manera estricta.
En la prác ti ca la Biblio te ca del Con gre so de los Es ta dos Unidos y la Biblioteca Nacional Británica han logrado un alto nivel de compatibilidad en los registros que intercambian, debido a que han trabajado en conjunto desde las primeras incursiones en el intercambio de registros bibliográficos.

Con esto, po dría es pe rar se que los pro ble mas de com pa ti bi li dad desparecerían ya que ambas bibliotecas manejan reglas comunes; por ejem plo, tan to la Biblio te ca Bri táni ca como la Biblio teca del Congreso utilizan la segunda edición de las Reglas de Catalogación Anglo Americanas. Sin embargo, debido a los problemas de interpretación y aplicación existentes en cada dependencia donde las reglas dejan cierto margen de interpretación, hasta la fecha no se ha logrado normalizar las formas de asien tos principales, en tre las dos or ganizaciones. Esto significa en la prác ti ca que cuan do se to man re gis tros de la base de da tos de la Biblio te ca del Con gre so y se in cor po ran a al gún ar chi vo de la Biblioteca Británica no existe garantía de que los asientos de nom bres de au to res per so na les o de en ti da des cor porati vas sean los mis mos, y con obje to de ase gu rar la con sis ten cia ca ta lo gráfi ca to dos los re gis tros exi gen ser ins pec ciona dos, as pec to que redunda en el costo-beneficio.

Se pue de de cir, en ge ne ral, que la nor ma li za ción de la es truc tu ra ló gi ca del re gis tro es un pre rre qui si to para su in ter cam bio, ya que es uno de los prime ros cri te rios para lo grarcom pa ti bilidad, en relación con esto los servicios de intercambio observan la Norma ISO 2709, por lo que el problema de la estructura lógica puede decirse que está resuelto.

Sin em bar go, el con te ni do de la es truc tu ra ló gi ca de pen de has ta el mo men to de los re que ri mien tos de la des crip ción ca ta lo grá fica de cada base de datos; esa descripción, se basa fundamentalmen te en las Re glas de Ca ta lo ga ción An gloameri ca nas 2 ó en las ISBD. Se es pe ra que es tas fuen tes de apo yo ca ta lo grá fi co me joren a corto plazo la normalización del contenido del registro.

En ge ne ral se pue de afir mar que la nor ma li za ción en el con tex to del intercambio de registros bibliográficos, es una herramienta que facilita la comunicación, reduce las variantes en muchas ocasiones innecesariasen los productos diseñados para cumplir objetivos similares.

Respecto al procesamiento automatizado de información, al registro bibliográfico se le ha considerado como un medio potencial eficiente de transferencia de información entre usuarios, ta les como usua rios pro duc to res de ba ses de da tos y sis te mas nacionalesoin ternacionales de al macena mien to y recuperación de in for mación, ser vicios dere sú menes ein dización, y produc to res de bibliografías, entre otros.

La exis ten cia de nor mas para cin tas de sie te y nue ve ca na les, ha resuelto innumerables dificultades en la transferencia de información a través de este soporte magnético.

Ade más de la di fícil ta rea de di se ñar y man te ner nor mas que provocan todas las posibles implementaciones, existe también el problema de su promoción y adopción. 
Las co sas se com pli can adi cio nal men te por la exis ten cia de diferen tes or ga nis mos que pro du cen nor mas que ope ran en di fe rentes niveles. Sin embargo, instituciones nacionales de productores de normas, tales como el American National Standards Institute, Inc. (ANSI), participa en la elaboración de normas a un nivel internacional y cada vez más juegan un papel primordial, organismos internacionales como la UNESCO, UNISIST, la Federación Internacional de Asociaciones de Bibliotecas (IFLA), todos ellos en colaboración con ISO quien es en la actualidad la responsable de la normalización internacional.

\section{Algunas Conclusiones.}

Hoy en día y en un marco internacional, se puede apreciar que exis ten va ria dos for ma tos de in tercam bio de in for ma ción bi bliográfica automatizada entre los que se destacan: MARC, UNIMARC, IN TER MARC, el Ma nual de Re fe ren cias del UNISIST, y elCCF, en tre otros. Se pue de de cir que la exis ten cia de di fe rentes for ma tos, re sul ta un per jui cio cos to so ya que im pli ca ge ne rar los pro gra mas de cóm pu to para con ver tir los da tos de un for ma to a otro cuan do se re quie re in ter cam biar in for ma ción, este as pec to reprime el intercambio de información bibliográfica debido a que la redacción de programas de cómputo, exigen de tiempo y dinero.

El in ter cam bio de in for ma ción en tre ba ses de da tos, es un fe nóme no que cada día ad quie re ma yor sen ti do, de bi do fun da men tal men te a que a par tir del in ter cam bio se lo gra en tre otros as pec tos el incremento en la normalización de registros bibliográficos al adop tar nor mas in ter na cio na les, aho rro en re cur sos hu ma nos dedi ca dos al pro ce sa mien to téc ni co de ma te ria les, aho rro en tiempos de procesamiento de documentos, y por lo tanto ahorro financiero, y además se cumple una de las funciones de las bibliotecas en lo que respecta a compartir sus recursos con otras unidades de información.

Al ser estructurado por el UNISIST y la UNESCO el Formato CCF (For ma to Co mún de Co mu ni ca ción), se afir mó que éste sería la nor ma in ter na cio nal que per mi ti ría el in ter cam bio de in formación entre diferentes sistemas con distintos formatos. Esto pue de serfac ti ble si se pre vee des de el di se ño de ba ses de da tos la adop ción del CCF, como for ma to de al ma ce na mien to y de in tercambio.

Sin em bar go, las ba ses de da tos que se en cuen tran en ope ra ción con base en al gún for ma to lo cal o in ter na cio nal, pre vio al CCF, como lo es el MARC, deberán ade cuar su for ma to si uno de sus objetivos es la compatibilidadcon otras ba ses de da tos que usen for ma to $\mathrm{CCF}$, con el fin de in ter cam biar su in for ma ción, as pec to que no siempre redundará positivamente en el costo-beneficio

En la actualidad las experiencias de compatibilidad de información que se re gis tran son las de los sis te mas INIS y AGRIS, que utilizan el mismo formato de almacenamiento e intercambio de re gis tros, así como las de los sis te mas BLAI SE y el de la Bi blioteca del Congreso, quienes utilizan los formatos UKMARC y
USMARC, respectivamente, ambos fundamentados en los formatos MARC.

La compatibilidad con el formato $\mathrm{CCF}$, requiere de programas sofisticados de conversión, se debe tener presente que, cuanto ma yor sea la di ver gen cia en tre for ma tos y la in for ma ción pro cesa da, ma yor será la di fi cul tad en la ela bo ra ción de los pro gra mas de conversión.

En el manual CCF, se aclara que no pretende ser un manual de co di fi ca ción y ca ta log a ción. Sin em bar go, la ex pe rien cia en México con el formato MARC, ha llevado a la generación de manuales cada vez más específicos para la utilización e in ter pre tación delfor mato, as pec to que eli mi na mu chas posibili da des de error en la apli ca ción de los ele men tos de co di fi ca ción y su relación con las áreas catalográficas.

El grupo Adhoc del formato CCF, debería tomar en cuenta esta po si bi li dad ya que si sólo se que da a ni vel de guía, oca sio na rá la ge ne ra ción de di fe ren tes ver sio nes CCF, as pec to que pue de desviarel obje ti vo principaldel for ma to: que pre ten de seruniversal.

Se de be rá te ner muy pre sen te, que el ma nual CCF, no es la so lución a la totalidad de actividades y de problemas técnicos que ocasiona la adopción de la automatización en bibliotecas y en cen tros de do cu men ta ción y que ade más exis ten po cas aplica ciones documentadas del mismo.

Se ría muy be né fi co que el gru po Adhoc del for ma to CCF, con side ra ra la po si bi li dad de ge ne rar un pa que te de pro gra mas de uso ge neral, estogaran tizaría, en parte, el uso uni versaldel for ma to.

Ojalá que este formato no sea sólo un nuevo intento de unificación bibliográfica ya que estudiando su estructura se puede observar que se orienta hacia una nueva filosofía en el diseño de bases de datos, aspecto que podría redundar en un manejo más eficaz de la información.

Res pec to al for ma to MARC, se pue de de cir que es en el que mayor ex pe rien cia exis te, en cuan to a su apli ca ción a ni vel in ter nacio nal. En Mé xi co, tam bién se ob ser va este fe nó me no, se po dría afirmar que la experiencia más profunda de los bibliotecarios me xi ca nos, en cuan to al uso y ma ne jo de for ma tos in ter na cionales, radica en el formato MARC, este aspecto lo demuestran las diferen tes ba ses de da tos me xi ca nas que se en cuen tran en ope ración, des de hace al gu nos años y que ba san su al ma ce na mien to de información en el formato MARC. Por lo que respecta al uso y aplicación del formato $\mathrm{CCF}$, los bibliotecarios mexicanos, se han in tro du ci do en su ma ne jo y apli ca ción a par tir de los úl ti mos dos años.

Por otra parte, se puede observar que el formato MARC, va en es tre cho de sa rro llo y apli ca ción con los nue vos avan ces tec no lógicos, muestra de ello son sus más recientes aplicaciones en CDROM y en el de sa rrollo del sis te ma ex per to MARC para ca taloga do res, sis te ma que se fun da men ta en los prin ci pios de in te ligencia artificial. 
Por otra parte, el formato CCF ha sido adoptado por el CONACYT, como norma para la creación de bases de datos, así como para el in tercambiodere gis tros bibliográficos en tre sis temas.

La adop ción del for ma to CCF en Mé xi co, pue de ser buen prin cipio para las instituciones que deseen desarrollar bases de datos automatizados.Por lo tanto, sería muy conveniente que se definan los cam pos mí ni mos CCF que de be rían ser usa dos en Mé xico. Esa de fi ni ción po dría es tar ba sa da en los ti pos de bi blio te cas y cen tros de do cu men ta ción me xi ca nos y esto au na do a los ni veles en el análisis de la información que se hace en ellos.

Se ría res pon sa bi li dad de la Bi blio te ca Na cio nal de Mé xi co, manejar en su totalidad el registro bibliográfico, aspecto que no puede suceder con otras instituciones debido a que persiguen dis tin tos ob je ti vos. Por tan to, la adop ción del for ma to CCF en su for ma más am plia co rres pon de ría a la bi blio te ca men cio na da ya que es ella quien de be ría fun gir como fuen te ca ta lo grá fica na cional.
A pesar de la exis ten cia de di ver sas ba ses de da tos bibliográficas mexicanas, no existe hasta el momentointercambioderegistros so bre al gún so por te mag nético en tre biblio te cas mexicanas, ni a ni vel na cio nal ni in ter na cio nal, más bien los for ma tos MARC y $\mathrm{CCF}$, se han usa do has ta el mo men to como base para la co di fi cación y almacenamiento de información. Sin embargo, el uso de esos for ma tos faci li ta rán en un fu tu ro la nor ma li za ción y el in tercambio de registros, aspecto que redundará en una mayor integración y cooperación entre las bibliotecas mexicanas.

Tam bién se pue de ob ser var que en paí ses la ti no ame ri ca nos tampocoexisten ex periencias documentadas sobre elintercambiode registros bibliográficos entre unidades de información. Sería muy interesante investigar las causas que impiden este tipo de cooperación. Normalización y cooperación son constantes que de ben te ner pre sen tes los or ganizado res y difu sores de in for ma ción de bi do al au men to de la pro duc ción de la mis ma, ante la necesidad de disminuir costos y de compartir el trabajo en búsqueda de mejores niveles de producción. Se debe comprender que si se deseasatisfacer las necesidades de información de los usua rios es pre ci so fo men tar el in tercam bio de proce sos, productos y servicios.

\section{BIBLIOGRAFIA}

ATTERTON, PAULINA. Manual para sistemas y servicios de información. París : UNESCO, 1978. 520 p.

AU BRAC, R. AGRIS—problems de compatabilitéetd'interconnection.En:Information systems: theirinterconnectionandcompatibility. Vienna, International Atomic energy Agency, pp. 35-40, 1975.

AVRAM, H. D. Recomendations towards the es ta blish ment of a common ex chan ge for mat for use by all agencies in the information community. En: Toward a common exchange format?

Procedings of the International Symposium on bibliographic Exchange Formats, Taormina, Sicily, 27-29 April, 1978 Budapest, OMKDK-Technoinform, pp. 135-146, 1978.

El servicio MARC : su historia y sus consecuencias.

México : Biblioteca Benjamín Franklin, 1980. 50 p.

BA BEL, DE BO RAH B. Archi val tape pro ces sing: con si de ra tions for a net work.En: Technical Services Quarterly. 4(1) Fall, pp. 11$18,1986$.

BA KER,D.F.Hermonization of national policies to im pro ve in tercon nec tion and compatibility. En: Infor ma tion sys tems: theirinterconnection and compatibility. Vienna, International Atomic Energy Agency, pp. 247-255, 1975.

BARNES, DA VID. Tape transfer special. En: Communication Technology Impact. 8(7) Oct., pp. 1-4, 1986.

BOIKO, N.V.; POLOZHENTSEVA, G. I. Compatibility of formats for representing bibliographic information in automated IRS's. Automaticdocumentationandmathematicallinguistics New Yorkm Vol. 14, No. 3, 1980, pp. 31- 40. trans la tion of 14, No. 5, pp. 17-25, 1980.

BOOKS, K. M. The online transfer of machine-rea dable data: a Pandora's box. En:Database (Wes ton, CT), Vol. 5, No. 1, pp. 18-21, 1982.

BROWN, T.P.Commu nications stan dards for on line in terchan ge of li brary in for ma tion.En: Li brary trends (Cham paign, IL), Vol. 31, No. 2, pp. 251-263, 1982. 
BRYHN, PER MORTEN. Exchange of Nordic bibliographic records. En: Catalogue \& Index. (81) Summer, pp. 6-8 tables, 1986.

BUFFET, P. Ex chan ge of bi blio gra phic data: pro blems rai sed by the ca rrier. En: Toward a coommon biblio gra phic exchan geformat? Proceedings of the International Symposium on Bibliographic Exchange Formats. Taormina, Sicily, 27-29 April. 1978. Budapest, OMKDK-Te chnoin form, pp. 21-24,1978. CAD WA LLA DER, G. Format Compatibilityand con versionamong bibliographic data bases. Philadelphia, Auerbach Corporation, 16 p. 1969.

DAHLBERG, I. Toward es tablishment of compatibility bet ween in de xing languages. En:Internationalclassification. (Mu nich), Vol. 8, No. 2, pp. 86-91, 1981.

DIERICKX, H. A proposed common format for existing and projected computerized bibliographic information systems in the Caribbean. Paris, Unesco, IV, 78 p. 1982.

DIE RICKX, H. AND HOPKIN SON, A. Toward a commonbibliographic exchangeformat? Proce edings of the In ternational Symposium onbibliographic Exchangeformats, Taormina, Sicily, 27-29Abril, 1978. Bu da pest, OMKDK-Technoin form, 214 p., 1978.

DUCHESNE, R. M. MARC and SUPERMARC. En: The ex chan ge ofbiblio gra phic data and the MARC for mat. Mu nich, We lag Dokumentation, pp. 37-57. 1972.

DUSOULIER, N. Compatibilité et normalisation. En: Information et documentation (Paris), Vol. 4, Desember, pp. 41-46. 1975.

FRENCH, E. J. Stan dar di za tion as a fac tor in in for ma tion trans fer. En: Journal ofinformation science (Ams ter dam), Vol. 3 No. 2, pp. 91-100. 1981

GOVE, N. ; et al.¿ Use of ISO stan dards in INIS. En: Informationsystems: theirinterconnectionand compatibility. Vien na, In ter na tional Atomic Energy Agency, pp. 335-447. 1975.

HEY MANS, F. How hu man- u sable is in ter chan gea ble? Or, shall we pro du ce ca ta lo gues or bibliographic-towers? En: Li brary re sources and technical services. (Chicago), Vol. 26, No. 2, pp. 156-169. 1982.

HITZEROTH, C. MADOK - The German magnetic tape ex chan ge for mat in the field of do cu men tation. En: Toward a common bibliographic exchange format? Proceedings of the International Symposium on bibliographic Exchange formats, Taormina, sicily, 27-29 April Budapest, 1978.

ISO standards handbook. Information transfer. Second edition, Geneva, International Organization for Standarization, 1982.

LEWIS, P. R. recent UNIMARC developments, British Library, London. En: IFLA Journal. 12(4), pp. 297-298, 1986.

LIBRARY SYSTEMS and Services Inc., and The Li brary Cor poration. Pri vate fi les on vi deodisc and CD- ROM. En: Library Sys tems Newsletter. 5(7) July, pp. 53-55, 1985.

LIM, HUCK TEE. Bibliographicinterchange/coordination in Southeast Asia.En:InternationalCataloguing. 16(1) Jan/Mar, pp. 4-6, 1987.

LYN NE M., NEU FELD AND MAR THA COR NOG. Da ta ba se his tory : from di no sau rus to com pact discs. En: Jour nal of the Ame rican Society for Information Science. 37(4) pp. 183-190, 1986.

MACKENZIE OWEN, J. Format incompatibility and the exchange of bibliographic information: a comparative study. Paris, Unesco, IV, 60 p. 1976.

MALICONICO,S.M. The co or dination of bibliographic con trol.En:Towarda commonbiblio graphic exchan geformat? Proce edings of the International Sympo sium on Biblio graphic Exchange Formats, Taormina, Sicily, 27-29 april 1978. Bu dapest, OMKDKTechnoinform, pp. 61-67. 1978.

MANSON, PAT. Bibliographic record transfer using electronic mail: a new service from John Menzies Library Services LTD. En: Vine. (62) May., pp. 32-34, 1986.

MEEK, C.L. Compatibility. En: Encyclopedia of computer science. New York, Petrocelli/Charter, pp. 236-237. 1976. 
NO LAN, R. E.; WHIT NEY, V. K. M.Dataelementstandardization:anapproachwith-forwardcompatibility.Draftfinalreport. Phase 1. Vienna, Evaluation Research Corporation, II, 46 p. 1978.

- - D ata ele ment stan dar di za tion: a metho do logy for des cribing nu me ric data. En: Datafor scien ce and te chno logy: Pro ce edings of the SeventhInternationalCODATAConference, Kyoto, Japan, 8-11 October 1980. Ox ford, per ga mon Press, pp.535-539. 1981.

SIL VER MAN, B. W., AND WIL SON, J.D. A be ta- bi no mial mo del for li brary sur vey data.En: JournalofDocumentation. 43(2), June, pp. 112-124, 1987.

SIMMONS, PETER. Using CCF: The Common communication Format. En: Information Technology and Libraries. 5(4) Dec. pp. 285-306, illus., 9 refs., 1986.

TAMURA, KIYOKO. Bibliogra phic in for ma tion and da ta ba ses in Ja pan. National Cen terfor Scien ce In for ma tion sys tems (SIS) En: International Cataloguing. 15(3) July/Sept., pp. 30-31, 1986.

UNESCO. Manual para el tratamiento de información referencial. UNESCO, CEPAL, 1985. (VERSION PRELIMINAR).

VERO NA, E. A de ca de of IFLA's work on the stan dar di za tion of bi blio gra phic des crip tion. IFLA journal (Mu nich), Vol. 6, No. 3 pp. 216-233. 1980.

WOLF, DIE TER. Pre li mi nary fin dings of a ques tion nai re on na tio nal mag ne tic tape and onlineservices. En: IFLA Jour nal. 12(4), pp. 299-300, 1986.

The cu rrent ac ti vi ties of the IFLA In ter na tio nal MARC Pro gram me: In ter na tio nal MARCP ro ject - Deuts che Bi blio thek (IMP- DB). En: IFLA Journal. 12(4), pp. 298-299, 1986. 